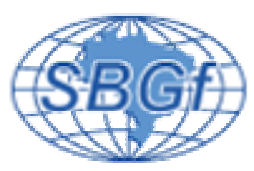

\title{
Estimativas dos teores de umidade utilizando o GPR no modo multi-offset. Um estudo na cidade de São Paulo/SP.
}

\begin{abstract}
${ }^{1}$ IAG-USP - Programa de pós-graduação, manuelle@iag.usp.br
Copyright 2004, SBGf - Sociedade Brasileira de Geofísica

Este texto foi preparado para a apresentação no I Simpósio de Geofísica da Sociedade Brasileira de Geofísica, São Paulo, 26-28 de setembro de 2004. Seu conteúdo foi revisado pela Comissão Tecno-científica do I SR-SBGf mas não necessariamente representa a opinião da SBGf ou de seus associados. E proibida necessariamente representa a opinião da SBGf ou de seus associados. E proibida a autorização da SBGf.
\end{abstract}

* Manuelle Santos Góis Paixão ${ }^{1}$, Renato Luiz Prado ${ }^{2}$, Liliana Alcazar Diogo ${ }^{2}$

2 IAG-USP - Departamento de Geofísica, renato@iag.usp.br, liliana@iag.usp.br

\section{Resumo}

São apresentadas estimativas dos teores de umidade do horizonte superficial do solo obtidas a partir de correlação empírica com as constantes dielétricas (Topp et al., 1980). As constantes dielétricas foram calculadas a partir dos valores das velocidades de propagação da onda direta. Foram adquiridos diversos perfis multi-offset em terreno típico de áreas tropicais e em períodos climáticos distintos. Em cada período de aquisição foram empregadas antenas de diferentes freqüências centrais (50 MHz, $100 \mathrm{MHz}$ e $200 \mathrm{MHz}$ ) e coletadas amostras de solo para a determinação dos seus teores de umidade. Os resultados obtidos a partir dos dados do GPR são bem coerentes com os das amostras de solos para os diferentes períodos investigados.

\section{Introdução e Objetivos}

A determinação de parâmetros hidrogeológicos como nível d'água e teor de umidade do solo tem uma ampla aplicação em estudos geoambientais. Através do método GPR - Ground Penetrating Radar, podemos estimar estes parâmetros, uma vez que as propriedades eletromagnéticas (e.g. $k$ : constante dielétrica) dos materiais de subsuperfície são severamente afetadas pela presença da água.

Alguns trabalhos já abordaram este assunto (Du \& Rummel, 1994; Huisman \& Bouten, 2001), mas em situações de solos homogêneos e com altas resistividades (geometria dos levantamentos - offset comum), obtendo as velocidades das ondas eletromagnéticas a partir das diferenças dos tempos de chegadas das ondas aéreas e diretas. Neste trabalho empregamos a técnica de multi-offset e em terreno típico de áreas tropicais, ou seja, onde as frações argila e silte contribuem de forma significativa na sua composição, tornando-o mais condutivo e, portanto, menos favorável ao emprego do GPR.

Como existem vários fatores que contribuem diretamente nos erros das medidas feitas com GPR, (e.g. deriva do instrumento e acoplamento da antena no solo durante aquisição), e estes não são facilmente quantificados, neste artigo abordamos uma sistemática que buscou minimizar os erros envolvidos no cálculo das velocidades (ajuste linear dos tempos de chegada das ondas diretas em cada conjunto multi-offset), analisando e comparando as diferenças das medidas observadas para o mesmo período e local, usando antenas de diferentes freqüências centrais ( $50 \mathrm{MHz}, 100 \mathrm{MHz}$ e $200 \mathrm{MHz}$ ).

Este trabalho trata de um dos aspectos do projeto de mestrado de um dos autores, intitulado "Análise da acurácia das estimativas de posicionamento do nível freático e dos teores de umidade do solo com o emprego dos métodos de sísmica de refração rasa e georadar a partir de um estudo no campus da USP, São Paulo/SP“.

\section{Metodologia e Problema Investigado}

Os ensaios foram realizados em 3 períodos distintos i.e. setembro de 2003, janeiro de 2004 e abril de 2004 que correspondem respectivamente aos períodos seco, chuvoso e intermediário em relação aos índices pluviométricos (setembro- $25 \mathrm{~mm}$; janeiro-203 mm; abril$80 \mathrm{~mm}$ ). As informações pluviométricas foram obtidas do banco de dados da Estação Meteorológica do IAG, situada no campus da USP, mesma região da realização dos ensaios geofísicos (Estação Meteorológica IAG, 2002).

A área de estudo está localizada na cidade de São Paulo, no Campus Armando de Salles Oliveira no CEPEUSP (Centro de Práticas Esportivas da Universidade de São Paulo) / Raia Olímpica, ao longo de um perfil linear de 20 $\mathrm{m}$ de comprimento, georeferenciado. Neste local ocorrem sedimentos aluviais quaternários sobrepostos aos sedimentos terciários da Formação Itaquaquecetuba (Riccomini, 1989).

Os dados foram adquiridos com o equipamento RAMAC da Mala Geosciences, com antenas de $50 \mathrm{MHz}, 100 \mathrm{MHz}$ e $200 \mathrm{MHz}$ (não blindadas). A aquisição foi feita ao longo do perfil georeferenciado de $20 \mathrm{~m}$, com emprego da geometria WARR - Wide Angle Reflection and Refraction, simulando a aquisição da sísmica de reflexão multicanal, ou seja, a antena transmissora permaneceu fixa e a receptora foi sendo deslocada de um offset mínimo ( $0 \mathrm{~m}$ para todas as antenas) até um offset máximo (2,9 m para as antenas de $100 \mathrm{MHz}$ e $200 \mathrm{MHz}$ e $5,9 \mathrm{~m}$ para a antena de $50 \mathrm{MHz}$ ) em intervalos de $0,1 \mathrm{~m}$ (antenas de $100 \mathrm{MHz}$ e $200 \mathrm{MHz}$ ) e 0,2 m (antena de 50 $\mathrm{MHz}$ ). O procedimento era repetido a cada nova posição da antena transmissora, que também se deslocou em intervalos de $0,1 \mathrm{~m}$ (antenas de $100 \mathrm{MHz}$ e $200 \mathrm{MHz}$ ) e $0,2 \mathrm{~m}$ (antena de $50 \mathrm{MHz}$ ). Etapas futuras do projeto permitirão o reordenamento dos traços em conjuntos CMP para se obter uma seção final empilhada.

Também foram feitos furos a trado e um poço para coleta de amostras para análises dos teores de umidade do solo à base de peso e volume (Klute,1986) para os 3 períodos 
Estimativas dos teores de umidade utilizando o GPR no modo multi-offset. Um estudo na cidade de São Paulo/SP.

distintos, sendo que a medição à base de volume (que exige a abertura de poço para coleta de amostra indeformada) ocorreu apenas na campanha de abril de 2004. As amostras foram coletadas de $0,5 \mathrm{~m}$ em $0,5 \mathrm{~m}$ até a profundidade aproximada de $3,5 \mathrm{~m}$ (referente ao nível d'água local, medido periodicamente em poço de monitoramento existente no local dos ensaios).

A partir desses dados também se obteve a coluna geológica (qualitativa) da linha geofísica (Figura 01).

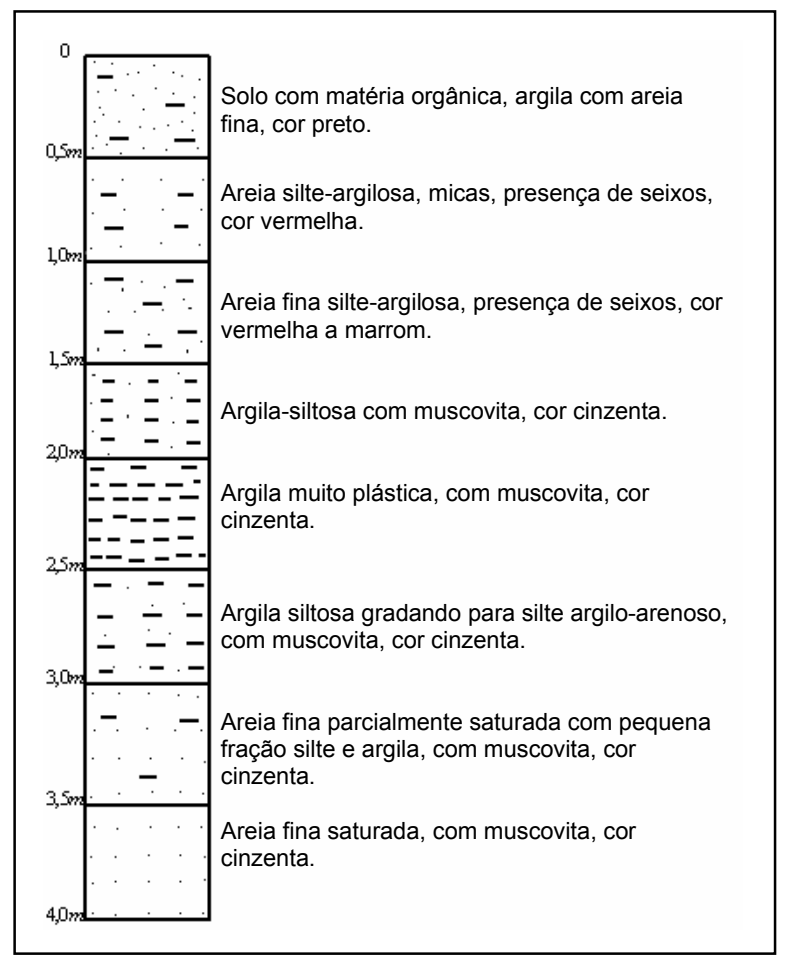

Figura 01 - Coluna geológica (descrição qualitativa) da linha geofísica (amostras do furo a trado)

Para analisar os radargramas e obter os tempos de chegada das ondas diretas utilizamos o aplicativo Seismic Unix - SU (Stockwell \& Cohen, 2000). Através do programa Gnuplot ${ }^{\circledR}$ (Williams \& Kelley, 2002) de livre distribuição, ajustamos os tempos obtidos a uma reta utilizando regressão linear com 7 repetições. A partir dos valores dos coeficientes angulares obtidos calculamos os valores das velocidades e os erros associados para cada conjunto WARR.

Como a velocidade das ondas de radar é obtida pela relação:

$$
v=c / \sqrt{k}
$$

onde: $c$ =velocidade da luz no vácuo $(0,3 \mathrm{~m} / \mathrm{ns})$ e $k=$ parte real da constante dielétrica (80 para água, 1 para o ar, 3 - 10 para sedimentos e solos secos e $30-$ 40 para argilas e siltes)

podemos obter também os respectivos valores de $k$.
A partir dos valores de $k$ calculados, utilizamos a relação empírica proposta por Topp et al. (1980) para obtermos os teores de umidade $\theta$.

$$
\theta=-5,3 \times 10^{-2}+2,92 \times 10^{-2} \times K-5,5 \times 10^{-4} \times K^{2}+4,3 \times 10^{-6} \times K^{3}
$$

Topp et al. (1980) concluem que a relação empírica dada pela equação 2 é válida para uma faixa de freqüências de $20 \mathrm{MHz}-1 \mathrm{GHz}$.

\section{Resultados}

Tanto para os dados de velocidade quanto para os de teor de umidade foi aplicada uma média móvel com período de 5 para suavizar as flutuações, mostrando com maior clareza o padrão ou a tendência dos dados, obtendo as seguintes respostas:

Para o mês de janeiro que choveu $203 \mathrm{~mm}$ as velocidades das ondas diretas obtidas com o emprego das diferentes antenas seguiram a tendência:

$v_{50 \mathrm{MHz}}>v_{100 \mathrm{MHz}}>v_{200 \mathrm{MHz}}$. O erro (aproximado) associado aos valores das velocidades foram $2,74 \%$, $3,14 \%$ e $3,29 \%$ respectivamente. Os teores de umidade calculados com base na relação empírica (equação 02) seguiram a seguinte tendência: $\theta_{200 \mathrm{MHz}}>\theta_{100 \mathrm{MHz}}>\theta_{50 \mathrm{MHz}}$ (Figura 02).

a)

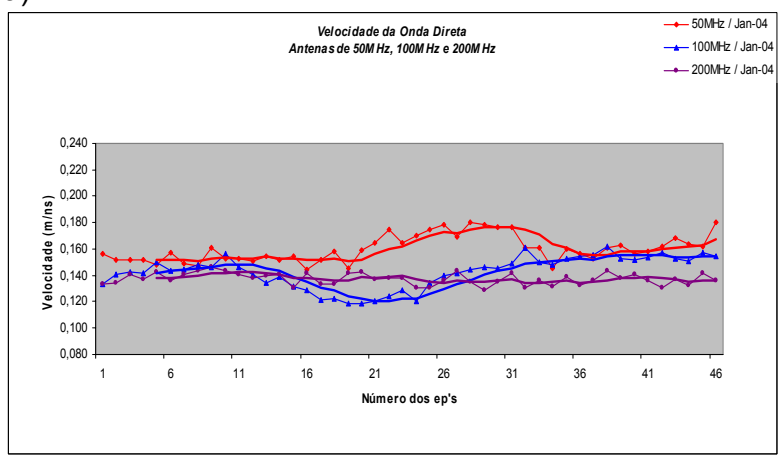

b)

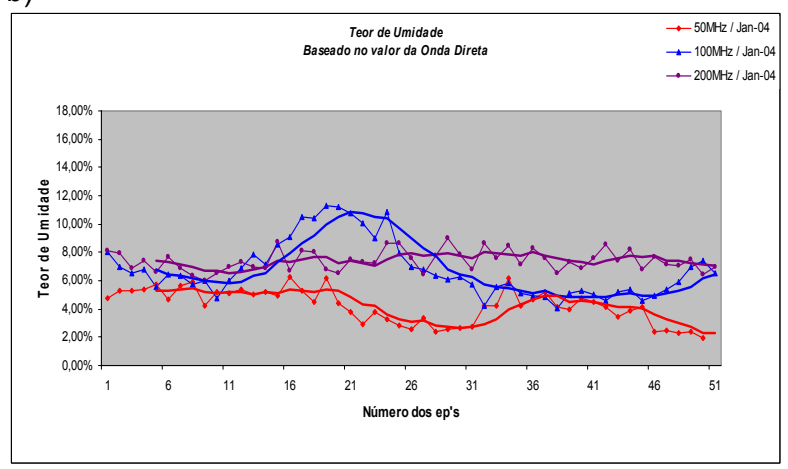

Figura 02 - a) Velocidade da onda direta das antenas de $50 \mathrm{MHz}, 100 \mathrm{MHz}$ e $200 \mathrm{MHz}$ para o mês de janeiro/04. b) Valores do teor de umidade das respectivas antenas para o mesmo período. 
No mês de abril, que choveu $80 \mathrm{~mm}$, as velocidades das ondas diretas obtidas com o emprego das diferentes antenas seguiram a tendência: $v_{50 \mathrm{MHz}}>v_{100 \mathrm{MHz}}>v_{200 \mathrm{MHz}}$. O erro associado aos valores das velocidades foram: $3,23 \%, 3,57 \%$ e $2,49 \%$ respectivamente (Figura 03).

a)

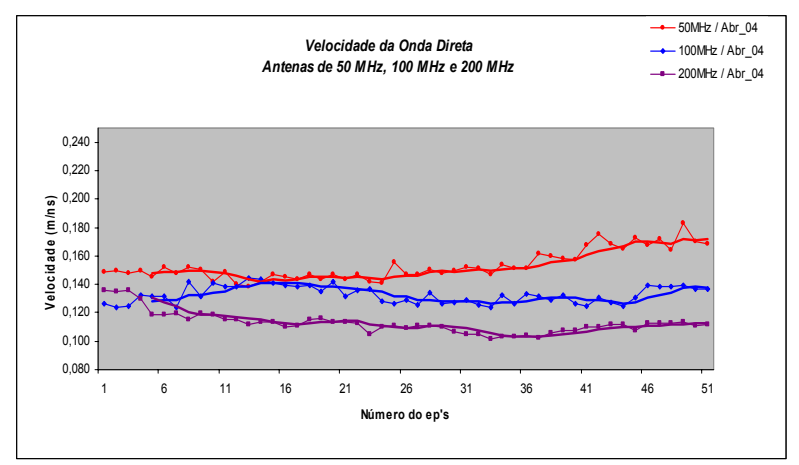

b)

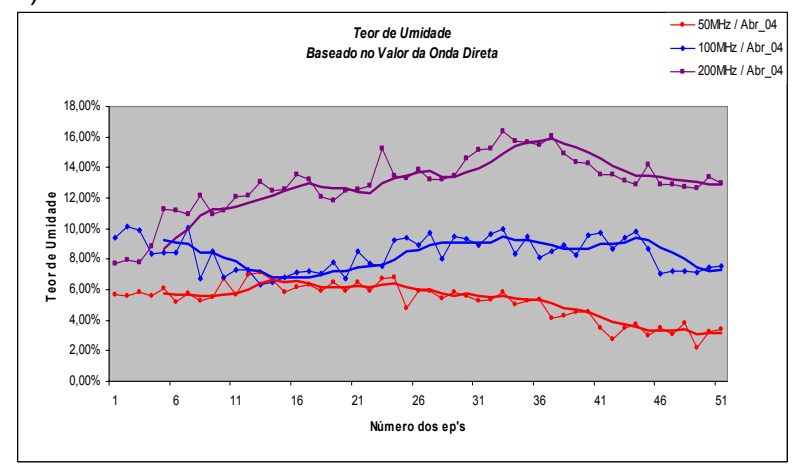

Figura 03 - a) Velocidades da onda direta das antenas de $50 \mathrm{MHz}, 100 \mathrm{MHz}$ e $200 \mathrm{MHz}$ para os mês de abril/04.

b) Valores do teor de umidade das respectivas antenas para o mesmo período.

No mês de setembro, que choveu $25 \mathrm{~mm}$, as velocidades da onda direta para a antena de $100 \mathrm{MHz}$ se diferenciam bastante das obtidas em janeiro/04 e abril/04, que por sua vez são muito próximas. O erro associado aos valores das velocidades em setembro/03 foi de 3,83\% (Figura 04a). a)

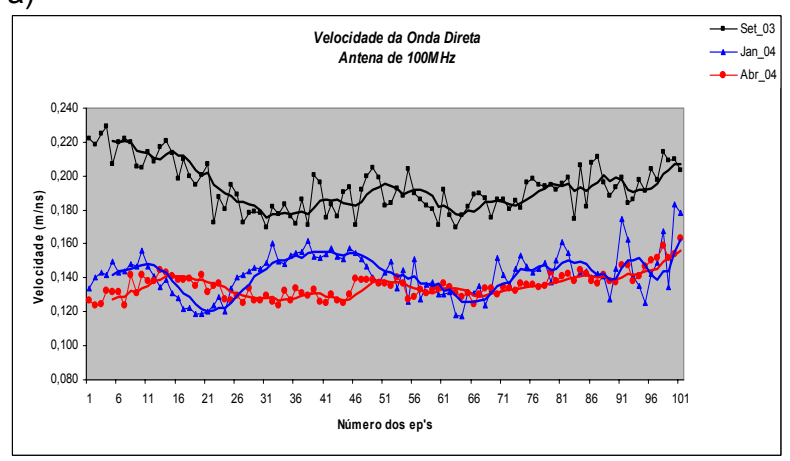

b)

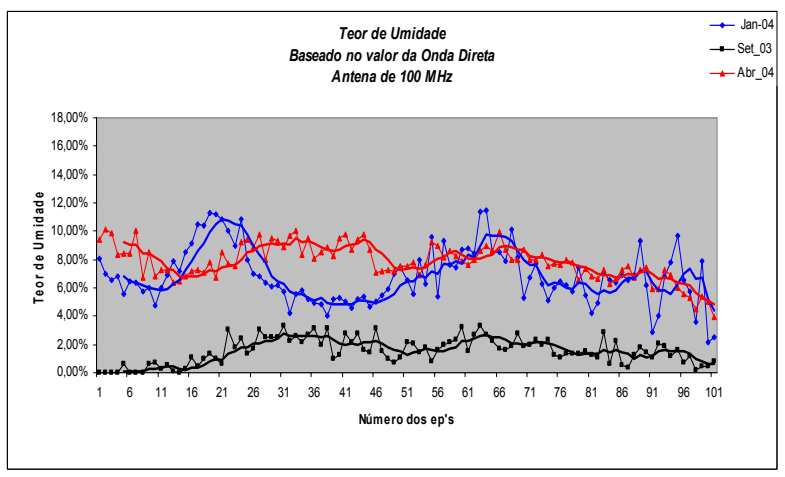

Figura 04 - a) Velocidades da onda direta da antena de $100 \mathrm{MHz}$ para os 3 períodos distintos (setembro/03, janeiro/04 e abril/04). b) Valores do teor de umidade da antena de $100 \mathrm{MHz}$ para os 3 períodos distintos (setembro/03, janeiro/04 e abril/04).

Com as amostras dos furos a trado obtivemos o teor de umidade a base de peso a cada $0,5 \mathrm{~m}$ nos 3 períodos distintos (Figura 05).

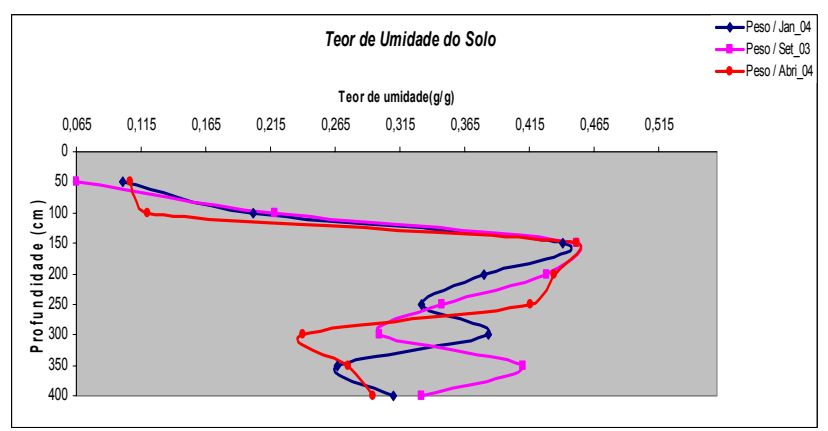

Figura 05 - Teores de umidade a base de peso para os meses de setembro/03, janeiro/04 e abril/04.

\section{Discussão e Conclusões}

De acordo com a equação 01 pode se observar que quanto maior o valor da constante dielétrica $k$, menor será o valor da velocidade. Poder-se-ia esperar, portanto, 
Estimativas dos teores de umidade utilizando o GPR no modo multi-offset. Um estudo na cidade de São Paulo/SP.

que em períodos mais chuvosos, como janeiro/04, as velocidades fossem menores do que as obtidas para o período intermediário de abril/04 e conseqüentemente obtivéssemos valores de umidades maiores para janeiro/04. Porém, não foi o que se observou tanto em relação aos dados do GPR quanto das amostras de solos, ou seja, os teores para os dois períodos são bastante próximos.

Uma provável explicação para este fato é que apesar do maior volume de chuva do mês de janeiro, as temperaturas médias deste período são bem superiores às de abril, favorecendo 0 processo de evapotranspiração e mantendo os teores de umidade dos horizontes mais superficiais $(<0,5 \mathrm{~m})$ muito próximos para os dois períodos.

Já em relação aos dados de setembro/03 (período seco) a velocidade da onda direta para antena de $100 \mathrm{MHz}$ é sensivelmente maior que as observadas em janeiro/04 e abril/04. Conseqüentemente, os teores de umidades obtidos são bem menores. Os dados de umidade obtidos das amostras de trado confirmam essa relação, ou seja, há realmente uma diminuição significativa nos teores de umidade para o mês de setembro/03 (Figura 05).

Os teores de umidade obtidos das amostras evidenciam que em profundidade os valores não se diferenciam muito para os 3 períodos investigados, excetuando-se o nível mais superficial, que em setembro apresenta valor bem inferior aos de janeiro e abril. Observa-se, também, que os teores obtidos com o GPR são mais próximos daqueles observados nas amostras do horizonte mais superficial $(0,5 \mathrm{~m})$. Portanto, todas as antenas parecem estar amostrando materiais do nível mais superficial do terreno, independentemente da sua freqüência central.

Essa observação é válida considerando o método empregado, ou seja, correlação obtida a partir da velocidade da onda direta no solo e concordam com observações de Galadera \& Parkin (2003), que realizaram testes com antenas de $100 \mathrm{MHz}$ e $450 \mathrm{MHz}$. Por outro lado, Clement e Ward (2003), apontam em seu trabalho que as antenas de menor freqüência propiciam a investigação de horizontes mais profundos (mesmo considerando o registro das ondas diretas), o que no presente estudo, e nas condições do ensaio, não parece ser verdadeiro.

Ainda que esses estudos se encontram em andamento e novos ensaios estão previstos para as próximas estações seca e chuvosa, podemos considerar que os valores dos teores de umidade obtidos a partir do GPR são bem coerentes, concordando com as variações sazonais de umidade observadas nas amostras. Diferenças pequenas, como as encontradas entre os períodos de janeiro e abril, não são sensíveis ao método.

\section{Agradecimentos}

À Direção do CEPEUSP; ao IAG/USP - Departamento de Geofísica; à Fapesp; à Secção Técnica de Serviços Meteorológicos/IAG - Prof. Dr. Ricardo Camargo e ao IPT - Laboratório de Física dos Solos.

\section{Referências}

Clement, W. P.; Ward, A.L., 2003. Using Ground Penetrating Radar to Measure Soil Moisture Content, American Society of Agricultural Engineering - ASAE, Annual Meeting, paper number 032275 $14 \mathrm{p}$.

Du, S., Rummel, P., 1994, Reconnaissance studies of moisture in the subsurface with GPR, Proceedings of the fifth international conference on Ground Penetrating Radar, GPR 94, pp1241-1248.

Huisman, J.A.; C. Sperl; W. Bouten; J.M. Verstraten, 2001, Soil water content measurements at different scales: accuracy of time domain reflectometry and ground-penetrating radar, Journal of Hydrology, 245,4858.

Galagedara, L. W.; Parkin, G. W., 2003, An Analysis of the GPR Direct Ground Wave Method for Soil Water Content Measurement, CGRG, Hydrological Processes, pp3615-3628.

Klute, A., 1986. Methods of soil analysis. Agronomy n.9, American Society of Agronomy. Wisconsin. 1188 p.

Observações de Superfície Efetuadas na Estação Meteorológica, 2002, v. 1, Secção Técnica de Serviços Meteorológicos- Instituto de Astronomia, Geofísica e Ciências Atmosféricas, Universidade de São Paulo, 16p.

Riccomini, C., 1989. Rift Continental do Sudeste do Brasil. Tese de Doutorado. IG/USP. São Paulo, 320p.

Stockwell, J.W.; Cohen, J. K., 2000, CWP/SU: Seismic Unix Release 33: A Free Package for Seismic Research And Processing. Center for Wave Phenomena, Colorado Scholl of Mines, Colorado.

Topp, G.C.; Davis, J.L.; Annan, A.P. 1980. Eletromagnetic determination of soil water content: measurements in coaxial transmission lines: Water Resources Research, 16, 574-582.

Williams, T.; Kelley, C., 2002, GNUPLOT, version 3.7. 